\title{
Pulmonary mucormycosis - diagnostic dilemma and uphill battle in treatment: A case report on life threatening fungal infection
}

\author{
${ }^{1}$ Sanchayan Thanancheyan, ${ }^{1}$ Aathavan Muthulingam, ${ }^{1}$ Nisanthan Selvaratnam, ${ }^{2}$ LSM Sigera, ${ }^{2}$ Primali Irosha \\ Jayasekera
}

${ }^{1}$ Teaching Hospital, Jaffna, Sri Lanka, ${ }^{2}$ Medical Research Institute, Colombo, Sri Lanka

Keywords: Invasive fungal infections, Mucormycetes, Pulmonary mucormycosis, Systemic antifungals, Immunodeficiency states, Amphotericine B

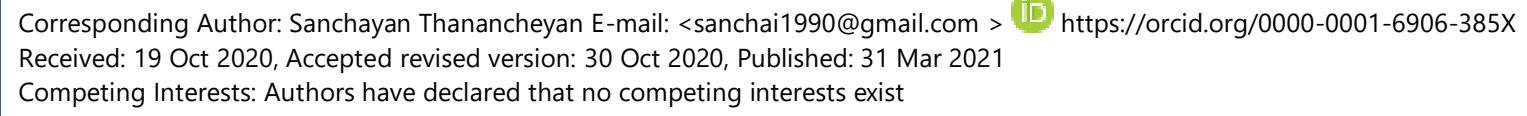

(C) Authors. This is an open-access article distributed under a Creative Commons Attribution-Share Alike 4.0 International License (CC BY-SA 4.0), which permits unrestricted use, distribution, and reproduction in any medium, provided the original author and source are attributed and materials are shared under the same license.

\section{Introduction}

Mucormycosis (earlier known as zygomycosis) is a serious but rare fungal infection caused by a group of moulds called mucormycetes. These are environmental fungi and mainly affect people with underlying immunodeficiency. The most common types that cause mucormycosis are Rhizopus species and Mucor species. Rhizomucor species, Syncephalastrum species, Cunninghamella bertholletiae, Apophysomyces, Lichtheimia, and Saksenaea are also implicated, but less commonly [1].

Mucormycosis is manifested by a variety of different syndromes in humans. Devastating rhinoorbital-cerebral and pulmonary infections are the most common syndromes [2]. However, any organ of human body could get affected [1]. There are many types of mucormycosis based on the sites of infection such as cutaneous, rhinocerebral, pulmonary, gastrointestinal, and disseminated [3]. Cutaneous mucormycosis (CM) occurs after the fungi enter the body through a break in the skin barrier. This is the most common form of mucormycosis in immunocompetent individuals. Rhinocerebral (RCM) mucormycosis and pulmonary mucormycosis (PM) are acquired by the inhalation of spores. RCM originates in the sinuses and can spread to the brain. PM occurs when spores reach alveoli and start to grow [2]. Gastrointestinal mucormycosis (GIM) is more common among young children than adults, especially in premature and low birth weight infants. Disseminated mucormycosis occurs when the infection spreads through the bloodstream and affects organs such as the brain, spleen, heart, and skin [1]. The fungi are angio-invasive and cause infarction of infected tissues [2].

Clinical manifestations of mucormycosis vary depending on the affected organs or systems. These wide spectra of symptoms include nasal ulceration, necrosis, periorbital swelling, 
impaired vision, ophthalmoplegia, sinusitis, headache, cranial neuropathies and coma (in RCM); cough, dyspnoea and haemoptysis (in PM); abdominal pain, hematemesis, perforation, peritonitis and bowel infarction (in GIM); and ulcers and skin necrosis (in CM) [2,3].

Almost all patients with invasive mucormycosis have some underlying pathological conditions. Diabetes mellitus (DM) (especially ketoacidotic state), glucocorticoid therapy, malignancies, hematopoietic cell transplantation and solid organ transplantation are commonly associated with mucormycosis. Use of deferoxamine, iron overload, AIDS, trauma/burns and malnutrition also seem to be risk factors. Voriconazole prophylaxis is found to be an independent risk factor in post-transplant patients, but the reason is not clear [2]. Still, some people develop mucormycosis in the absence of any identifiable risk factor [3].

Rhizopus species, one of the major implicated organisms in mucormycosis, thrive in ketoacidotoc conditions as they possess an enzyme called ketone reductase that makes them capable of utilizing ketones as an energy source [2]. They also prefer a high iron environment and deferoxamine-iron chelate (feroxamine) which act as a siderophore and increases the iron uptake by the fungus is believed to be the reason why mucormycosis is seen commonly in patients who underwent iron chelation using deferoxamine. Feroxamine abolishes the fungistatic effect of serum on Rhizopus and increases the growth of the fungus [4]. Statins are believed to have activity against a wide range of the agents of mucormycosis [2].

\section{Case presentation}

A 68-year-old man, with a background history of type 2 DM, was admitted to Teaching Hospital, Jaffna (THJ) with a complaint of insidious onset of worsening shortness of breath and right sided pleuritic type of chest pain over last five days. He also had a non-productive cough. There was no history of fever. He had loss of appetite and loss of weight of recent onset. $\mathrm{He}$ was an ex-smoker and ex-alcohol user. On examination, he was tachypnoeic but did not have signs of heart failure. On auscultation, fine crepitations heard over right middle and lower zone and left lower zone. Oxygen saturation $\left(\mathrm{SpO}_{2}\right)$ at room air was $95 \%$. All other system examinations were unremarkable. 12 lead Electrocardiography (ECG) was normal. Blood investigations were unremarkable. (Table 1 ) Chest $X$ ray $(C X R)$ showed consolidation in the right middle lobe with a normal cardiac silhouette. (Figure 1)

Table 1. Summary of investigations (excluding bronchoscopy investigations)

\begin{tabular}{|c|c|c|c|c|c|c|}
\hline \multirow[t]{2}{*}{ Investigations } & & \multicolumn{5}{|c|}{ Days since first admission } \\
\hline & $D_{1}$ & $D_{29}$ & $D_{37}$ & $D_{84}$ & $D_{89}$ & $D_{93}$ \\
\hline 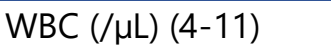 & 10.00 & 10.97 & 9.37 & 20.30 & 22.88 & - \\
\hline $\mathrm{N}(\%)$ & 68.6 & 73.9 & 68.0 & 86.1 & 92.0 & - \\
\hline L (\%) & 23.0 & 18.4 & 20.1 & 9.0 & 4.3 & - \\
\hline M (\%) & 6.6 & 5.3 & 9.2 & 4.7 & 3.5 & - \\
\hline E (\%) & 1.3 & 1.98 & 2.3 & 0.1 & 0.1 & - \\
\hline $\mathrm{Hb}(\mathrm{g} / \mathrm{dL})(11.0-16.0)$ & 13.7 & 12.4 & 10.9 & 10.4 & 9.2 & - \\
\hline PIt $(/ \mu \mathrm{L})(150-450)$ & 249 & 260 & 257 & 357 & 317 & - \\
\hline ALT (U/L) (16-63) & 26 & 17 & 19 & - & 20 & - \\
\hline AST (U/L) (15-37) & 19 & 09 & 16 & - & 17 & - \\
\hline $\operatorname{ALP}(\mathrm{U} / \mathrm{L})(46-116)$ & - & 76 & 60 & - & 161 & - \\
\hline TP (g/L) (64-82) & - & 82 & - & - & 66 & - \\
\hline
\end{tabular}




\begin{tabular}{|c|c|c|c|c|c|c|}
\hline $\begin{array}{l}\text { Albumin }(\mathrm{g} / \mathrm{L})(34-50) \\
\text { Globulin }(\mathrm{g} / \mathrm{L})(22-48)\end{array}$ & $\begin{array}{l}- \\
-\end{array}$ & $\begin{array}{l}29 \\
53\end{array}$ & - & - & $\begin{array}{l}14 \\
52\end{array}$ & - \\
\hline $\mathrm{Na}(\mathrm{mmol} / \mathrm{L})(136-145)$ & 139 & 139 & 134 & 131 & 137 & 139 \\
\hline $\mathrm{K}(\mathrm{mmol} / \mathrm{L})(3.5-5.1)$ & 4.8 & 4.6 & 3.5 & 4.7 & 4.1 & 3.4 \\
\hline $\mathrm{BU} \mathrm{mmol} / \mathrm{L}(2.5-6.4)$ & - & 3.2 & 2.8 & 7.5 & 26.3 & 12.1 \\
\hline $\mathrm{SCr} \mu \mathrm{mol} / \mathrm{L}(62-115)$ & 88 & 69 & 95 & 107 & 289 & 118 \\
\hline ESR $\mathrm{mm} / 1^{\text {st }}$ hour & - & 98 & - & 92 & 125 & - \\
\hline CRP (mg/L) (0-3) & 6 & 25 & 19 & 232 & 207 & 96 \\
\hline Blood picture $\left(D_{91}\right)$ & \multicolumn{6}{|c|}{$\begin{array}{l}\text { Reactive neutrophil leucocytosis; Normocytic anaemia with } \\
\text { marked rouleaux compatible with anaemia of chronic disease }\end{array}$} \\
\hline SPE $\left(D_{91}\right)$ & \multicolumn{6}{|c|}{ Normal pattern } \\
\hline Blood culture $\left(D_{34} \& D_{86}\right)$ & \multicolumn{6}{|c|}{ No growth } \\
\hline Urine culture $\left(D_{87}\right)$ & \multicolumn{6}{|c|}{ No growth } \\
\hline AGA & \multicolumn{6}{|l|}{ Negative } \\
\hline CT guided biopsy & \multicolumn{6}{|c|}{ Inflammatory lesion } \\
\hline Melioidosis antibody & \multicolumn{6}{|l|}{ Negative } \\
\hline
\end{tabular}

WBC, white blood cells; N, neutrophils; L, lymphocytes; E, eosinophils; Hb, haemoglobin; PIt, platelets; ALT, alanine transaminase; AST, aspartate transaminase; ALP, alkaline phosphatase; TP, total protein; Alb, albumin; Glo, globulin; S.cr, serum creatinine; $\mathrm{BU}$, blood urea; $\mathrm{Na}$, sodium; $\mathrm{K}$, potassium; $\mathrm{CRP}$, c-reactive protein; ESR, erythrocyte sedimentation rate; $\mathrm{SPE}$, serum protein electrophoresis; $\mathrm{AGA}$, Aspergillus galactomannan assay; $\mathrm{BAL}$, bronchoalveolar lavage; $\left(D_{x}\right)$, days since first admission

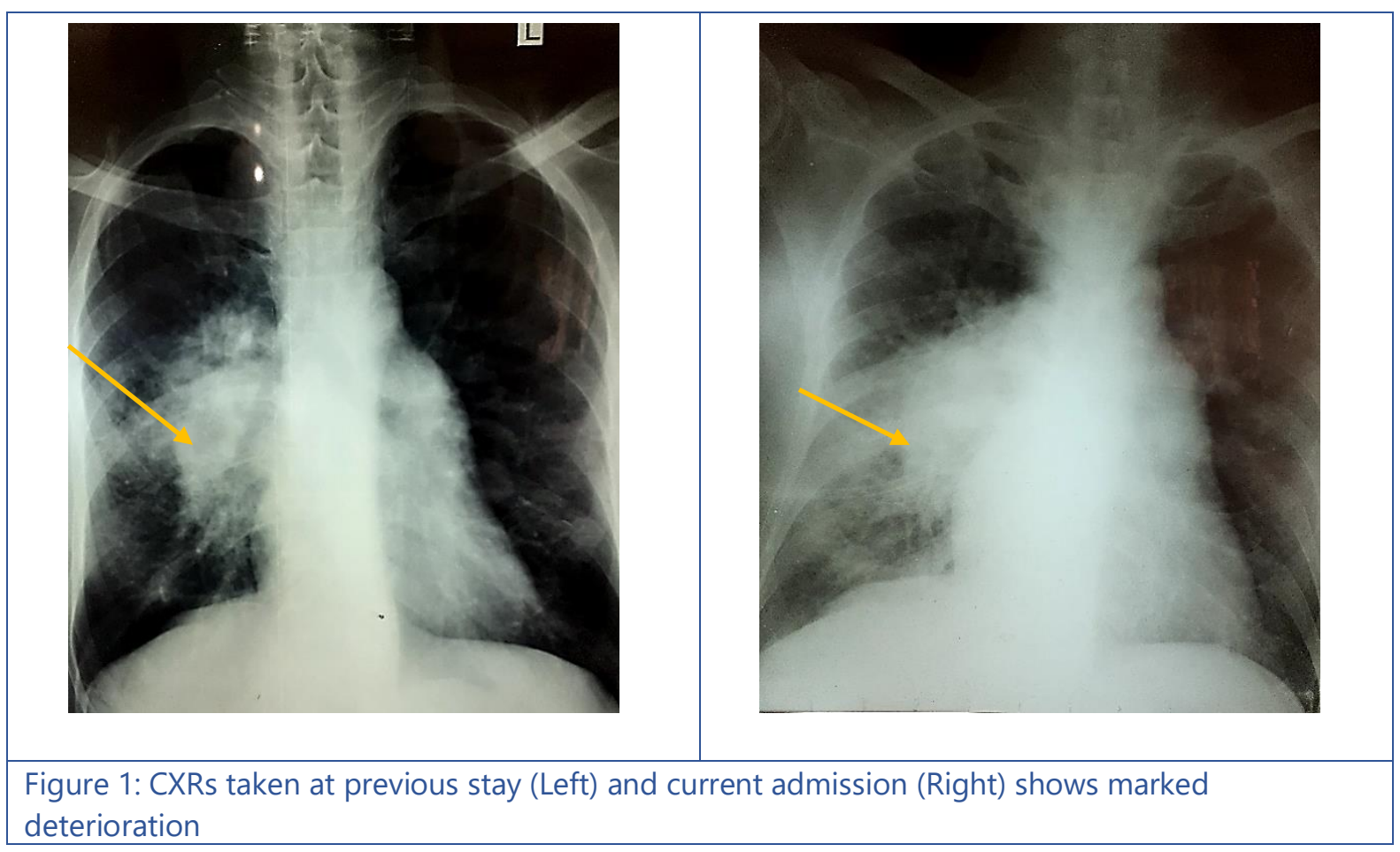

Ultrasonography of the abdomen showed grade I fatty liver. He underwent bronchoscopy with bronchoalveolar lavage (BAL) and samples were sent for cytology, cultures (pyogenic and tuberculosis) and GeneXpert. Cytology was negative for malignancy. Pyogenic culture returned a moderate growth of Pseudomonas and coliform. Tuberculosis (TB) culture and GeneXpert were negative. (Table 2). 
Table 2: Summary of bronchoscopic investigations

\begin{tabular}{|c|c|c|}
\hline Day & Bronchoscopic investigations & Result \\
\hline $\mathbf{D}_{2}$ & $\begin{array}{l}\text { BAL Cytology } \\
\text { Bacterial culture } \\
\text { TB culture } \\
\text { GeneXpert }\end{array}$ & $\begin{array}{l}\text { Negative for malignant cells } \\
\text { Moderate mixed growth of Pseudomonas species } \\
\& \text { coliform } \\
\text { Contaminated } \\
\text { Negative }\end{array}$ \\
\hline$D_{13}$ & $\begin{array}{l}\text { Bronchial mucosal biopsy histology } \\
\text { Bronchial brush cytology }\end{array}$ & $\begin{array}{l}\text { Granulation tissue with active inflammation } \\
\text { Negative for malignant cells }\end{array}$ \\
\hline $\mathbf{D}_{28}$ & $\begin{array}{l}\text { BAL Bacterial culture } \\
\text { Fungal culture } \\
\text { TB culture } \\
\text { GeneXpert MTB/RIF } \\
\text { Bronchial mucosal biopsy }\end{array}$ & $\begin{array}{l}\text { No growth } \\
\text { Growth of Aspergillus niger } \\
\text { No growth } \\
\text { Negative } \\
\text { Tissue with active inflammation }\end{array}$ \\
\hline$D_{87}$ & $\begin{array}{l}\text { BAL Bacterial culture } \\
\text { Fungal culture } \\
\text { DME } \\
\text { TB culture } \\
\text { GeneXpert MTB/RIF } \\
\text { Bronchial mucosal biopsy histology } \\
\text { DME } \\
\text { culture }\end{array}$ & $\begin{array}{l}\text { Streptococcus \& Enterococcus species isolated } \\
\text { Growth of Candida parapsilosis } \\
\text { Not done due to risk of covid-19 } \\
\text { No growth } \\
\text { Negative } \\
\text { Inflamed mucosa } \\
\text { Fungal filaments seen suggestive of Zygomycetes } \\
\text { Negative for fungi }\end{array}$ \\
\hline
\end{tabular}

$B A L$, bronchoalveolar lavage; TB, tuberculosis; $D M E$, direct microscopic examination; $\left(D_{x}\right)$, days since first admission

He was started on antibiotics. Contrast enhanced computed tomography (CECT) of chest and abdomen was done (Figure $2 \& 3$ ) which showed a right lower lobe apical segment tissue mass with enlarged pre and para tracheal lymph nodes.

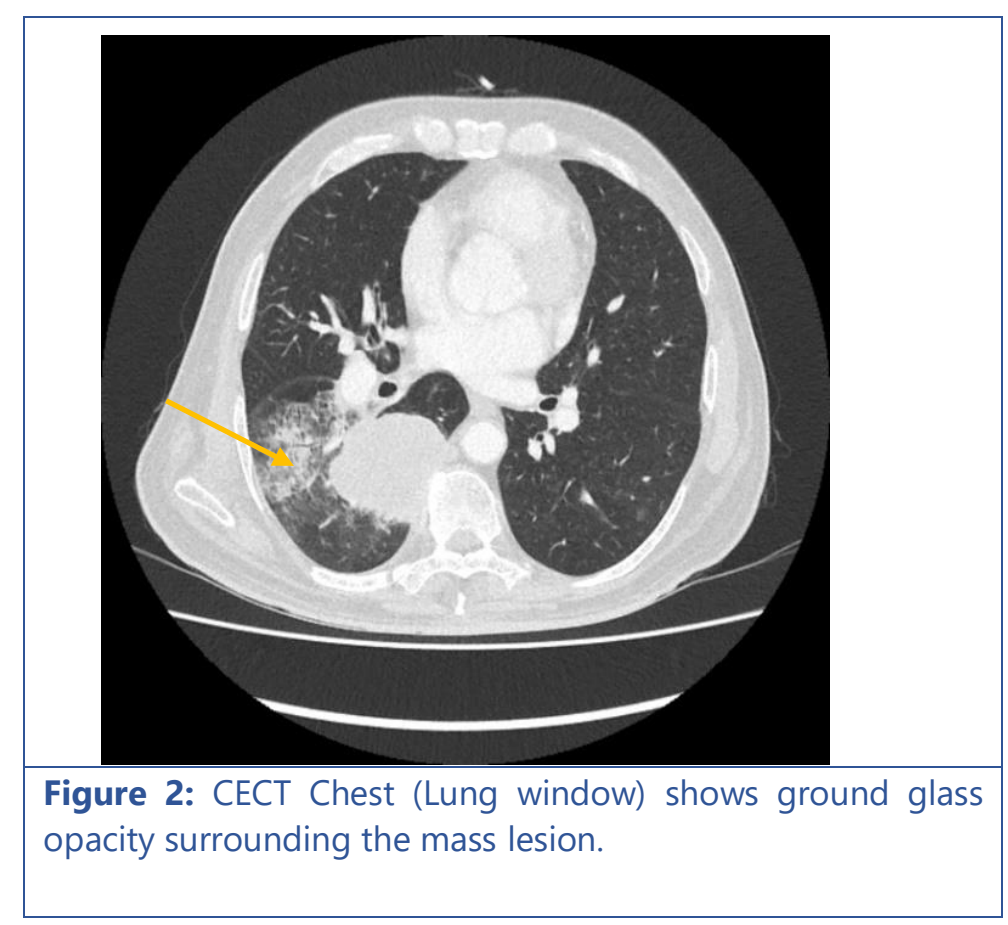




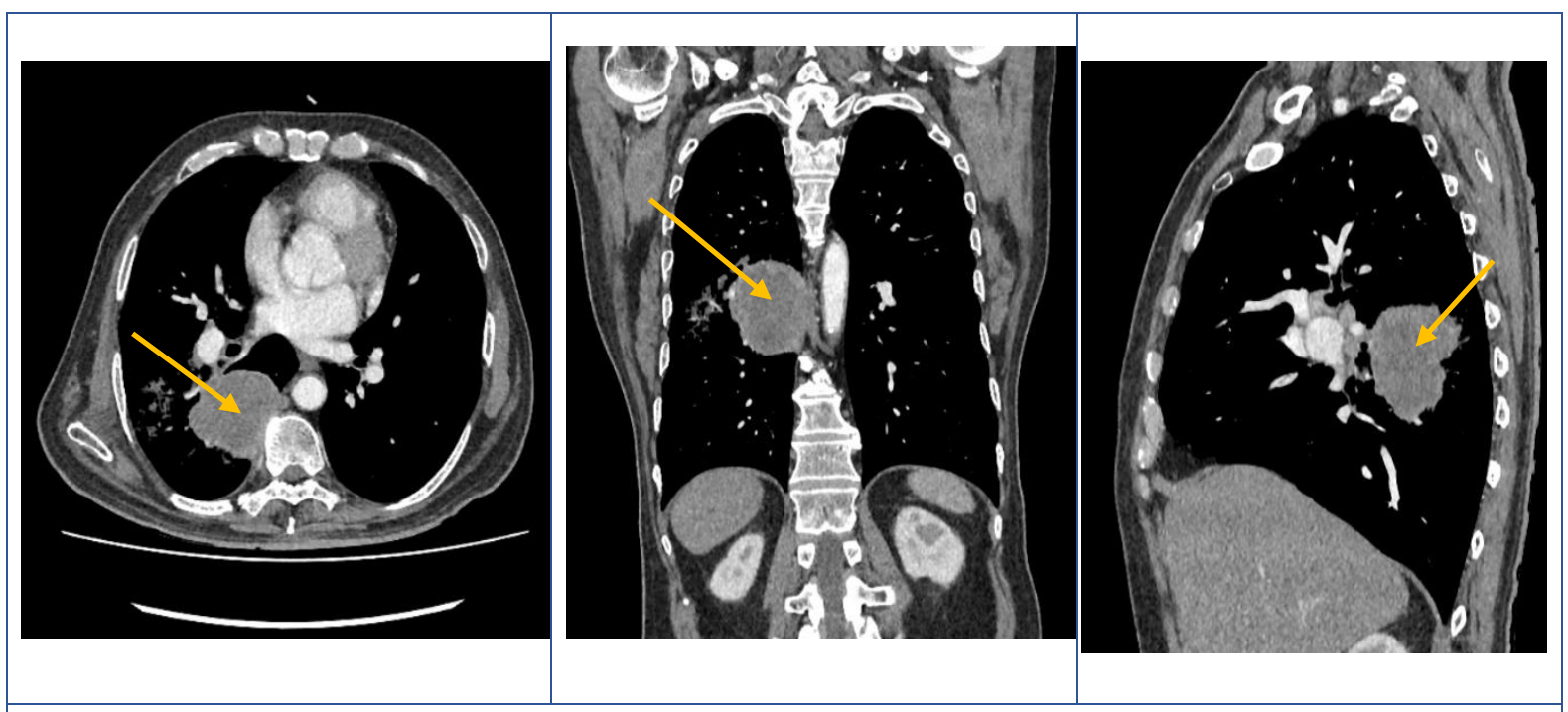

Figure 3: Axial (Left), Coronal (Middle) and Sagittal (Right) views of CECT Chest and Abdomen (Abdominal window) which shows growth of mucormycosis in left lung tissue

As a clinical diagnosis was yet to be made, he underwent another bronchoscopy with bronchial brush and biopsy. (Table 2) Bronchial biopsy was reported to be granulation tissue with active inflammation. Subsequently he underwent $C T$ guided biopsy of the lung mass and the histology turned out to be of an inflammatory lesion. He was discharged with oral antibiotics.

As the patient's clinical condition did not improve with antibiotics, he was admitted again and underwent bronchoscopy for the third time with BAL and bronchial biopsy. (Table 2) Growth of Aspergillus niger was detected in the cultures at THJ. Another biopsy sample was sent to the Medical Research Institute (MRI) in Colombo for opinion from a mycologist who also confirmed the growth of the same species. Aspergillus galactomannan assay was negative. Clinical diagnosis of invasive aspergillosis was made and the patient was started on voriconazole PO $400 \mathrm{mg}$ b.i.d on first day followed by $200 \mathrm{mg}$ b.i.d from second day onwards. He showed mild improvement of symptoms and was subsequently discharged from the hospital on voriconazole. Follow-up was arranged at the respiratory clinic at THJ.

During the follow-up, over the next one and a half months, patient was noted to be gradually deteriorating. His symptoms persisted and the repeat CXRs showed worsening of shadows. He was re-admitted to THJ for further evaluation. CXR on admission showed progressive pulmonary infiltrates (mass like consolidation). Blood investigation showed no signs of improvement of ongoing inflammation (Table 1). He was started on IV meropenem $1 \mathrm{~g}$ 8hourly and oral voriconazole was continued. He underwent another bronchoscopy with BAL and bronchial biopsy for cultures and histology with special stains for fungi. (Table 2) Samples were also sent to MRI for fungal studies. Inflamed mucosa was seen in the histology of biopsy while growth of Streptococcus and Enterococcus species were noted in BAL culture.

He became clinically unwell with increased work of breathing. His arterial blood gas (ABG) analysis showed evidence of hypercapnic respiratory failure and he was initiated on non- 
invasive ventilation (NIV). IV liposomal amphotericin B was started empirically in addition to voriconazole.

Meanwhile, growth of Candida parapsilosis was observed in the BAL sample at MRI. Fungal filaments suggestive of Zygomycetes were seen in direct microscopy of the bronchial biopsy (Figure 4) though the culture was negative for fungi. Voriconazole was omitted and treatment with amphotericin B was continued. Unfortunately, despite the best efforts of the medical team he continued to deteriorate and finally succumbed to illness.

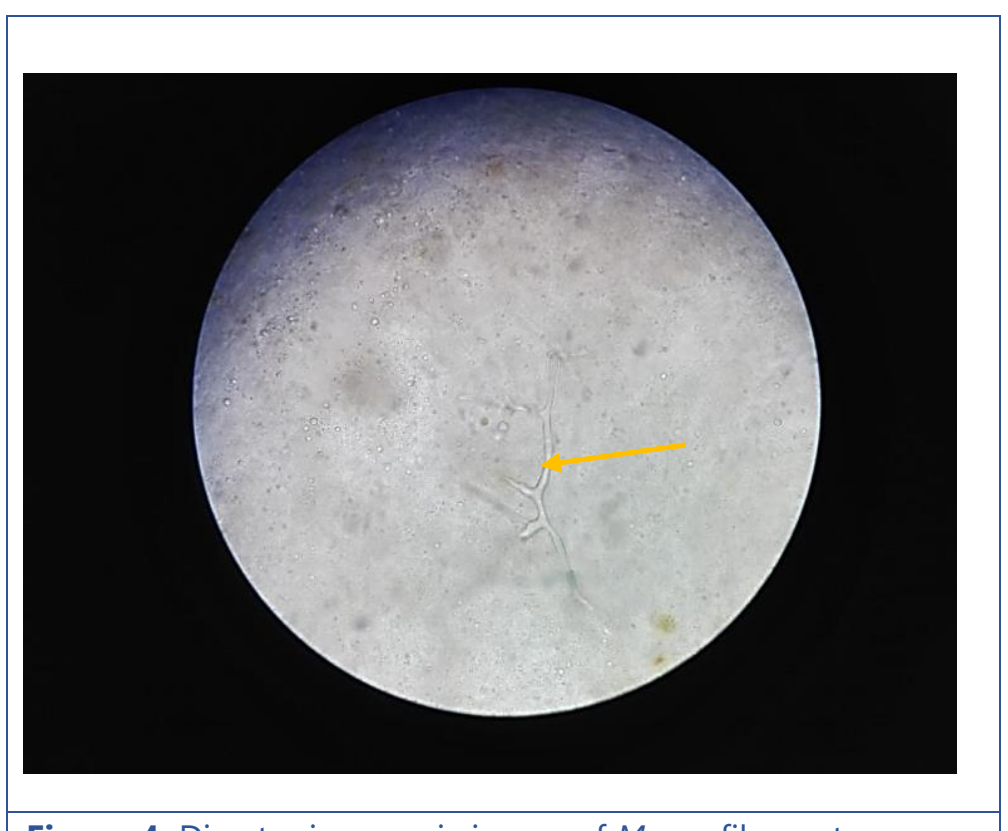

Figure 4: Direct microscopic image of Mucor filaments

\section{Discussion}

Pulmonary mucormycosis is a rapidly progressive infection. Spores of the Mucormycetes are larger than those of Aspergillus fumigatus [5], the common organism implicated in pulmonary aspergillosis [6], therefore they are commonly entrapped in the upper airway and sinuses. However, occasionally, they can bypass the upper airway and can be inhaled into the lower airway. Inhalation of spores into the bronchioles and alveoli leads to clinical symptoms of fever, dry cough, haemoptysis, chest pain, and shortness of breath [2,3] Pneumonia with pulmonary infarction and necrosis results and the infection can spread to the mediastinum and heart or disseminate via the blood stream to other organs. The most common associated conditions are haematologic malignancies, treatment with glucocorticoids or deferoxamine, and solid organ transplantation; it is less common in diabetics when compared to rhinocerebral mucormycosis [2]. This patient is a known patient with type $2 \mathrm{DM}$ with suboptimal glycaemic control. He did not have any other risk factors for PM.

The standard diagnosis of mucormycosis requires identification of the organisms in the tissues with culture confirmation. However, as the positivity rate of cultures are very low, histopathologic identification of an organism with a structure typical of mucorales could be considered as evidence of infection. Also, isolation of these fungi in culture does not necessarily prove infection because of the high chances of contamination. Microscopic 
evidence for PM is usually obtained from sputum or bronchoalveolar lavage (BAL) specimens. Broad, non-septate hyphae are characteristic and are often the first indicator of mucormycosis. Hyphae can also be demonstrated on lung biopsy. The diagnosis of pulmonary mucormycosis is difficult because of the non-availability of tissue for the demonstration of the organism and the similarity of clinical presentation with other invasive fungal infections. So, radiographic evidence $(C X R, C T)$ is often used to support the diagnosis [2].

Difficulties in diagnosing PM are evident from this case history. Initial positivity for Aspergillus niger was most probably due to contamination as these species are not commonly implicated in the pathogenesis of pulmonary aspergillosis (PA) [6] and given the fact that the clinical condition of the patient did not improve with treatment with voriconazole which is the drug of choice in the setting of PA. This contamination paved the way for the misdiagnosis of PA. Growth of Candida parapsilosis, observed in the last bronchoscopic sample, is also most probably a contamination as this fungus is typically a commensal of human skin [7]. Even though this organism can cause disseminated disease, the incidence of true Candida pneumonia is considerably low despite its frequent isolation from BAL [8]. It should be also noted that voriconazole, with which this patient was treated for a considerably long duration, has very good activity against Candida parapsilosis [7]. Identification of Pseudomonas, coliform, Streptococcus and Enterococcus in bronchoscopic cultures is also most probably due to contamination as these organisms are commensals in humans and the patient's clinical condition did not improve with broad spectrum IV antibiotics [9].

Air-space consolidations, cavitary lesions and the halo sign are relatively uncommon among patients with PM. Multiple nodules $(\geqslant 10)$ and micronodules (those $<1 \mathrm{~cm}$ in size) are more commonly seen. Pleural effusions are also more frequently observed among patients with PZ than among those with invasive pulmonary aspergillosis (IPA) [10] Even though the halo sign (ground-glass attenuation surrounding a nodule) is characteristic of angioinvasive fungi, clinically it is not helpful as both PM and IPA cause this radiographic change. Reversed halo sign, a focal area of ground-glass attenuation surrounded by a ring of consolidation, has also been reported in PM. PM appears to be the most common condition to cause the reversed halo sign in immunocompromised hosts [2]. CXR of this patient showed right sided consolidation with surrounding ground glass shadows with few peripheral nodules. Repeated CXR showed gradual worsening. HRCT showed a right sided mass lesion with ground glass attenuation surrounding it. Pleural effusion, cavitary lesions or halo signs were not noted.

Beta-D-glucan (BDG) is an antigen found in the cell wall of most fungal species including Candida, Aspergillus and Pneumocystis [11]. Galactomannan is a polysaccharide which is found primarily in the cell walls of Aspergillus species [12]. Mucorales do not have these cell wall antigens. Thus serum and BAL BDG and the Aspergillus galactomannan assay (AGA) could be used to exclude other invasive fungal infections in clinical practice [2]. Serum AGA sensitivity is significantly lower than that of BAL [11]. During the first admission, AGA was found to be negative in this patient. Nevertheless, the low sensitivity of AGA coupled with the finding of Aspergillus in the BAL put the accuracy of the result into question and treatment for IA was started. 
The utility of polymerase chain reaction (PCR)-based techniques on histology specimens is being studied and the results are promising. But this technique is not widely used as yet. Matrix-assisted laser desorption ionization-time of flight (MALDI-TOF) mass spectrometry can be used to identify the causative species from cultures [2].

Treatment of mucormycosis involves a combination of surgical and pharmacological therapy. Elimination or treatment of underlying immunosuppression is necessary. Aggressive surgical debridement of involved tissues should be considered. Intravenous (IV) amphotericin B (lipid formulation), started at $5 \mathrm{mg} / \mathrm{kg}$ daily and increased to $10 \mathrm{mg} / \mathrm{kg}$ daily, is the drug of choice for initial therapy. Early initiation of antifungal therapy improves the outcome of infection with mucormycosis [2].

Once the patient responds well to initial therapy, posaconazole or isavuconazole can be used for oral step-down therapy. Amphotericin B should be continued until the patient has shown signs of improvement which will usually take several weeks. In patients who do not respond to amphotericin B, posaconazole or isavuconazole are used as alternatives. Other antifungal agents, including voriconazole, fluconazole and flucytosine, are not effective against the Mucorales. Although the echinocandins are not used in clinical practice to treat mucormycosis, they could be useful as $R$. oryzae - the most common cause of mucormycosis - expresses the target enzyme for echinocandins. Combination therapy is not recommended. Therapy should continue until there is clinical and radiographic resolution of active disease. It usually takes months and some patients remain on therapy for life [2].

The prognosis for mucormycosis, except cutaneous mucormycosis, is poor, even with optimal management. Independent risk factors for mortality include disseminated infection, renal failure and infection with Cunninghamella species. The mortality rate in patients with pulmonary mucormycosis is higher (87\%) than for patients with rhino-orbital-cerebral involvement (25-62\%). Widely disseminated mucormycosis carries a mortality rate of $90 \%$ to $100 \%$ [2].

\section{Acknowledgements}

We thank the house officers and nursing staff who played an important role in managing this patient. We are also grateful to Dr. G. Rajanthi, Consultant Microbiologist, THJ, for her efforts to establish the diagnosis.

\section{References}

1. Fungal diseases. (2019, May 28). Retrieved September 23, 2020, from Centers for Disease Control and Prevention: https://www.cdc.gov/fungal/diseases/mucormycosis/index.html

2. Cox GM, Kaufman CA, Bond S. Mucormycosis (zygomycosis). UpToDate. Retrieved September 27, 2020, from Mucormycosis (zygomycosis) - UpToDate

3. Rare Disease Database - Mucormycosis. (2018). Retrieved September 26, 2020, from NORD - National Organization for Rare Diseases: https://rarediseases.org/rarediseases/mucormycosis 
4. Boelaert JR, de Locht M, Van Cutsem J, Kerrels V, Cantinieaux B, Verdonck A, Van Landuyt HW, Schneider YJ. Mucormycosis during deferoxamine therapy is a siderophore-mediated infection. In vitro and in vivo animal studies. The Journal of clinical investigation. 1993 May 1;91(5):1979-86. https://doi.org/10.1172/JCI116419

5. Chamilos G, Lewis RE, Hu J, Xiao L, Zal T, Gilliet M, Halder G, Kontoyiannis DP. Drosophila melanogaster as a model host to dissect the immunopathogenesis of zygomycosis. Proceedings of the National Academy of Sciences. $2008 \mathrm{Jul}$ 8;105(27):9367-72. https://doi.org/10.1073/pnas.0709578105

6. Person AK, Chudgar SM, Norton BL, Tong BC, Stout JE. Aspergillus niger: an unusual cause of invasive pulmonary aspergillosis. Journal of Medical Microbiology. 2010 Jul;59(Pt 7):834. https://doi.org/10.1099/jmm.0.018309-0

7. Trofa D, Gácser A, Nosanchuk JD. Candida parapsilosis, an emerging fungal pathogen. Clinical microbiology reviews. 2008 Oct 1;21(4):606-25. https://doi.org/10.1128/CMR.00013-08

8. Pendleton KM, Huffnagle GB, Dickson RP. The significance of Candida in the human respiratory tract: our evolving understanding. Pathogens and Disease. 2017 Apr;75(3): ftx029. https://doi.org/10.1093/femspd/ftx029

9. LaMorte, W. W. (2016, May 04). Infectious agents. Retrieved October 18, 2020, from Boston University School of Public Health: https://sphweb.bumc.bu.edu/otlt/mphmodules/ph/ph709_infectiousagents/PH709_InfectiousAgents3.html

10. Chamilos G, Marom EM, Lewis RE, Lionakis MS, Kontoyiannis DP. Predictors of pulmonary zygomycosis versus invasive pulmonary aspergillosis in patients with cancer. Clinical Infectious Diseases. 2005 Jul 1;41(1):60-6. https://doi.org/10.1086/430710

11. Theel ES, Doern CD. Point-counterpoint: $\beta$-d-Glucan testing is important for diagnosis of invasive fungal infections. Journal of clinical microbiology. 2013 Nov 1;51(11):347883. https://doi.org/10.1128/JCM.01737-13

12. Zhou W, Li H, Zhang Y, Huang M, He Q, Li P, Zhang F, Shi Y, Su X. Diagnostic value of galactomannan antigen test in serum and bronchoalveolar lavage fluid samples from patients with nonneutropenic invasive pulmonary aspergillosis. Journal of Clinical Microbiology. 2017 Jul 1;55(7):2153-61. https://doi.org/10.1128/JCM.00345-17 